as do catalytic activation energy and electric resistivity. A wave-mechanical theory of hardness, based on the more or less total completion of the BRILLouri-zones, is proposed.

\section{Uber den verschiedenen Einfluß fett- oder ölhaltiger Nahrung auf den Steringehalt der Nebenniere}

Die in den letzten Jahren erkannte Zugehörigkeit der Sexual- und Nebennierenrindenhormone zu den Sterinen erhöht das Interesse für den Sterinstoffwechsel im allgemeinen und für den Sterinhaushalt einiger endokriner Drüsen im speziellen. Bereits vor der Isolierung der Rindenhormone wurde die Beteiligung der Nebenniere am Cholesterinstoffwechsel diskutiert und dieses Organ als eine zentrale Stelle der Bildung oder Speicherung des Körpercholesterins angesehen. Es war auch bekannt, da $\beta$ bei Schädigungen toxischer, infektiöser Natur, sowie bei Hunger, Hitze-, Kälteeinwirkung und dergleichen die Nebenniere sehr bald einen größeren Teil ihres Cholesterinvorrates verliert. Dagegen sind die Umstände, welche zu einer Erhöhung des Steringehaltes der Nebenniere führen, noch wenig erforscht. Insbesondere ist das Ausgangsmaterial der Sterinbildung nicht abgeklärt. Sowohl Eiweiß, wie Fett und Kohlehydrat, aber auch niedermolekulare Stoffwechselzwischenprodulte mit 2 bis $3 \mathrm{C}$-Atomen wurden in Betracht gezogen'.

In Versuchen an weißen Ratten konnte festgestellt werden, daß es auf diätetischem Wege möglich ist, eine Vermehrung der Nebennierensterine herbeizuführen. Unter den zahlreich benutzten Futtermischungen bewährte sich bis jetzt am besten die Ergänzung einer kohlehydratreichen Diät durch physiologische Mengen pflanzlicher Öle. Ein Ersatz des Öls durch gleiche Mengen Fett ergab viel niederere Sterinwerte in der Rattennebenniere.

a) Durchschnittlicher Cholesteringehalt der beiden Nebennieren bei Verfüterung ölhaltiger Nahrung (24 Tiere) $5,67 \%$ entsprechend einem Absolutgehalt ron $2,17 \mathrm{mg}$ Cholesterin.

b) Durchschnittlicher Cholesteringehalt der beiden Nebeunieren hei Verfütterung einer sonst gleich zusammengesetzten, aber fetthaltigen Diät (21 Tiere) $=2,60^{\circ}$. . Dies entspricht einer durchschnittlichen Cholesterinmenge von $0,88 \mathrm{mg}$

Die mit ölhaltigem Futter crährten Tiere haben somit mebr als doppelt so hohe Cholesterinmengen in der Nebenniere.

Beispiel einer Futtermischung; $40 \mathrm{~g}$ Kartoffeln; $20 \mathrm{~g}$ Brot; $20 \mathrm{~g}$ Mais; $10 \mathrm{~g}$ Vollmilchpulver; $10 \mathrm{~g}$ Ol bzw. Fett. Danehen rohe und gekochte Vegetabilien. Fütterungsdauer 10-14 Tage.

Die Uberlegenheit der Öle gegenuiber den Fetten in bezug auf die Cholesterinanhäufung in der Nebenniere ist wohl dem höheren Gehalt derselben an den ungesättigten Fettsäuren (Öl-, Linol-, Linolensäure) zuzuschreiben. $\mathrm{Ob}$ diese OOle das direkte Baumaterial für die Cholesterinsynthese darstellen (wofür manche Literaturangabe sprechen würde'), oder ob das $\mathrm{O}_{1}$ bloß die Ansammlung der Sterins begiinstigt, ist noch nicht entschieden. Beachtenswert ist, daB eine derartige Anreicherung der Ncbenniere an Cholesterin von einer $\mathrm{Zu}$ nahme des Körpergewichtes, von einem besseren Aussehen und von einer erhöhten Widerstandsfähigkeit der Tiere operativen Fingriffen gegenüber begleitet ist.

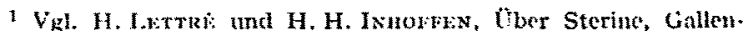
siuren und verwandte Nahrstoffe, Stuttgart 1980. - W. R. B.oor, Biochemistry of the fatty acids and other compounds, the lipicis, New York 1943.
Umgekehrt ist bekannt, dab bei geringem Cholesteringehalt der Nebenniere Tier und Mensch nicht selten geschwächt erscheinen. Gelten die ungesättigten Fettsäuren als unentbehrliche Nährstoffe mit Schutzwirkung gegenüber mancher Schädigung der Haut und der inneren Organe, so scheint ihnen darüber hinaus eine Bedeutung fiir den Sterin- (und wahrscheinlich für den Hormon-) umsatz des Körpers zuzukommen.

\section{Summary}

White rats fed on diets containing oils have much more sterols in their adrenals than control rats in whose diets the oils have been substituted by fats.

\section{ABELIN}

Medizinisch-chemisches Institut und "Hallerianum" der Universität Bern, den 9. Februar 1946.

\section{Elektronenmikroskopische Untersuchungen an Zellulosefasern nach Behandlung mit Ultraschall}

Bei der direkten Untersuchung des submikroskopischen Baues von Zellulosefasern besteht die Hauptschwierigkeit darin, so feine Faserbruchstiicke mit unveränderter, natürlicher Textur zu erhalten, daB sie im Elektronenmikroskop leicht durchstrahlt werden können. Bisher wurde diese Faserzerteilung mittels Schwingmahlung ${ }^{\mathfrak{l}}$ vorgenommen oder durch Zerquetschen stark hydrolysierter und gequollener Fasern versucht ${ }^{2}$. Diese Verfahren führten zu elektronenmikroskopisch durchsichtigen Präparaten, aber das Zellulosegerüst war derart zerstört, daß die übermikroskopischen Bilder nur mit großen Schwierigkeiten interpretiert und kaum mit den übrigen experimentellen Kenntnissen über den Faserfeinbau in Ubereinstimmung gebracht werden konnten. Vor allem fehlte auch der bildliche Zusammenhang zwischen mikroskopischen und submikroskopischen $\mathrm{Fa}$ serbausteinen.

Wir haben gefunden, daß bei Behandlung der Fasern in starken Uliraschallfeldern eine sehr feine und schonende Zerteilung eintritt, wobei die ursprungliche Textur der abgespaltenen Faserteile vollständig erhalten bleibt. Es ist ferner leicht, den Übergang von mikroskopisch sichtbaren Spaltstiucken zu den elektronenmikroskopisch abgebildeten Molekülsträngen darzustellen, so daß die Interpretation der übermikraskopischen Bilder sichergestellt wird.

Natürliche Zellulosefasern (Ramie, Hanf, Baumwolle etc.) zeigen bereits nach Beschallung während 3-10 Minuten eine außerordentlich feine Fibrillenaufspaltung (Abb. 1). Es zeigt sich, da $B$ nur die gröbsten der abgespaltenen Fäserchen mikroskopisch aufgelöst werden können (Phasenkontrastverfahren!), ihre feineren Enden verlieren sich im submikroskopischen Gebiete. Innerhalb des mikroskopischen Auflösungsbereichs ergibt sich ein gleichmäßiger Größenübergang der Fibrillendurchmesser von sehr groben Spaltstücken (uber $10 \mu$ breit) bis zu den feinsten, optisch auflösbaren Teilchen. Auch diese letzteren besitzen noch einen ungestörten Feinbau, denn ihre Doppelbrechung ist durchaus normal. Daraus geht hervor, daB in allen untersuchten Pflanzenfasern in mikroskopischen Bereiche keine vorgebildeten Bausteine einheitlicher GröBenordnung vorhanden sind.

1 W. Wercin, Koll. Z. 98,131 (1942).

2 F. Husemasw, J. makromolek. Chem., 3. Reihe 1,16, 108 (1943). 